\title{
Evaluation of financial sustainability of the Japanese medical system: Analysis of the length of hospital stay for diabetes patients
}

\author{
$\underline{\text { Nawata, K. }}{ }^{1}$ and K. Kawabuchi ${ }^{2}$ \\ ${ }^{1}$ Graduate School of Engineering, University of Tokyo, Bunkyo-ku, Tokyo 113-8656, Japan \\ ${ }^{2}$ Graduate School of Medical and Dental Sciences,, Tokyo Medical and Dental University, Bunkyo-ku, Tokyo, \\ 113-8510, Japan \\ Email:nawata@tmi.t.u-tokyo.ac.jp
}

\begin{abstract}
As Japan's population continues to age, the total medical expenditure is rapidly increasing, becoming a serious financial concern. In fiscal year 2012, the medical expenditure was 39.217 trillion yen, compromising $8.30 \%$ of the GDP (Ministry of Health, Labour and Welfare, 2014). Since Japan instituted a mandatory public health insurance system in 1961, the public expenditure and the amount of health insurance premiums reached 15.146 trillion and 19.120 trillion yen, respectively. The direct payment by patients was just 4.619 trillion yen or just $11.9 \%$ of the total medical expenditure. Hence, the financial sustainability of the system is now a very serious question, and controlling medical expenditures by limiting the length of stay (LOS) in hospital has become a controversial issue. In this paper, we evaluate the LOS of diabetes patients. The medical expenditure for diabetes was 1,209 billion yen, compromising about 3\% of the total medical expenditure, and diabetes was the third-most costly disease, after malignant tumor (cancer, 3,327 billion yen) and renal insufficiency (1,490 billion yen). Moreover, various researchers have pointed out that comorbid diabetes increases the risk of fatality and prolongs LOS, such that the true cost of diabetes might be much higher than the direct one. A large part of the medical cost of diabetic patients is determined by the LOS. However, the LOS for diabetes patients has not been widely studied, and only a few studies have been done in Japan.
\end{abstract}

In this paper, we analyze the LOS of type 2 diabetes patients by the Box-Cox transformation model (BC MLE) when variances of error terms vary among different hospitals. The maximum likelihood estimator (BC MLE) which maximizes the likelihood function under the normality assumption is used for the estimation of the BC model. However, Showalter (1994) reported large biases of the BC MLE when heterogeneity exists in variances. Heteroscedasticity is a very important problem in the $\mathrm{BC}$ model, as mentioned even in the original paper of Box and Cox (1964). For LOS in particular, variances are often very different among hospitals even after controlling for the characteristics of diseases, treatments and patients. We propose a new estimator which is consistent even under heteroscedasticity. We then analyze the LOS of type 2 diabetes patients using the data set of 1,571 patients collected from 17 general hospitals in Japan by the proposed method. Finally, we evaluate the financial effects on the Japanese medical system based on the obtained results. In Japan, a new inclusive payment system based on the Diagnosis Procedure Combination (DPC) was introduced in April 2003, and the system is now referred as the DPC/PDPS (per diem payment system). As of April 2014, a total of 1,585 hospitals had joined the DPC system and an additional 278 hospitals were preparing to join the DPS/PDPS (hereafter DPC hospitals). The DPC hospitals comprise about $25 \%$ of the 7,483 general hospitals in Japan. The DPC hospitals have 511,439 beds (474,981 beds for already joined hospitals and 36,458 for soon-to-join hospitals), which represents $57 \%$ of the total number of beds $(897,749$ beds) of all general hospitals. The DPC hospitals are required to computerize their medical information, and thus it has become possible for us to use large-scale data sets consisting of many hospitals and patients.

The variables found to affect the LOS were the purposes of hospitalization, numbers of secondary diseases and complications, acute hospitalization, and principal disease E117. We found surprisingly large differences in LOS among hospitals, even after eliminating the influence of patient characteristics and principal disease classifications. There were also large differences among estimates of the variances, and the feasibility of the proposed model was strongly suggested. We finally evaluated the effects of shortening the LOS and found that as much as 50 billion yen could be saved by reducing LOS for type 2 diabetes patients.

Keywords: Cox-Box transformation, heteroscedasticity, length of hospital stay, diabetes 
Nawata and Kawabuchi, Evaluation of financial sustainability of the Japanese medical system: Analysis of the length of hospital stay for diabetes patients

\section{INTRODUCTION}

As Japan's population ages, the country's total medical expenditure has been rapidly increasing, becoming a serious financial concern. According to the Ministry of Health, Labour and Welfare (2014), the medical expenditure was 39.217 trillion yen, comprising $8.30 \%$ of the Gross Domestic Product (GDP) in fiscal year 2012. In fiscal year 2002, the medical expenditure of Japan was 30.418 trillion yen; that is, the medical expenditure has increased by 9.699 trillion yen or $23.1 \%$ in these 10 years despite the fact that the annual nominal GDP did not increase but rather decreased in that period (510.8 trillion yen in fiscal year 2002 and 472.6 trillion yen in fiscal year 2012). Since Japan instituted the mandatory public health insurance system and all Japanese have been required to join some types of public insurance, the public expenditure and the amount of health insurance premium have reached 15.146 trillion and 19.120 trillion yen, respectively. The direct payment by patients was just 4.619 trillion yen or just $11.9 \%$ of the total medical expenditure. Hence, the financial sustainability of the system is now a very serious question, and controlling the medical expenditure by limiting the length of stay (LOS) in hospital has become a controversial issue. In this paper, we evaluate the LOS of type 2 diabetes patients. Unlike type 1 diabetes, which primarily involves genetic factors, type 2 diabetes can be prevented and controlled by improving the lifestyles of patients. The medical expenditure on diabetes in 1961 was 1,209 billion yen, compromising about $3 \%$ of the total medical expenditure; diabetes was the third-most costly disease, after malignant tumor (cancer, 3,327 billion yen) and renal insufficiency (1,490 billion yen). Moreover, Kudo et al. (2011) and Inoue et al. (2006) have pointed out that having diabetes as a comorbidity prolonged LOS, and the true cost of diabetes may be higher than the above number. A large part of the medical cost of diabetic patients is determined by LOS. However, LOS for diabetic patients has not been widely studied, and only few studies have been done in Japan (Mutou et al. 1999, Soumiya et al. 2004, and Nawata and Kawabuchi 2015).

In this paper, we analyze LOS in hospital by the Box-Cox transformation model (BC model), taking account for the different variances among hospitals. For details of the BC model, see Sakia (1992) and Hossain (2011). The maximum likelihood estimator (BC MLE), which maximizes the likelihood function under the normality assumption, is used for the estimation of the BC model. However, Showalter (1994) reported large biases of the BC MLE when heterogeneity exists in variances. Heteroscedasticity is a very important problem in the BC model, as stated even in the original paper of Box and Cox (1964). For LOS in particular, variances often differ greatly among hospitals, even after controlling for the characteristics of diseases, treatments and patients. We propose a new estimator that functions well even under heteroscedasticity.

In Japan, a new inclusive payment system based on the Diagnosis Procedure Combination (DPC) was introduced in April 2003, and the system is now referred as the DPC/PDPS (per diem payment system). As of April 2014, a total of 1,585 hospitals had joined the DPC system and an additional 278 hospitals were preparing to join the DPS/PDPS (hereafter DPC hospitals). The DPC hospitals comprise about $25 \%$ of the 7,483 general hospitals in Japan. The DPC hospitals have 511,439 beds $(474,981$ beds for already-joined hospitals and 36,458 for preparing-to-join hospitals), which represents $57 \%$ of the total number of beds ( 897,749 beds) in all general hospitals. The DPC hospitals are required to computerize their medical information, which makes it possible for us to use large-scale data sets.

We then analyze the LOS of type 2 diabetes patients using the DPC data set of 1,571 patients collected from 17 general hospitals in Japan by the proposed estimator. Finally, we evaluate the financial effects of shortening LOS.

\section{MODELS}

\subsection{BC model and BC MLE}

Suppose that the LOS of the patient $j$ in the hospital $i$ is given by the $\mathrm{BC}$ model:

$$
\begin{aligned}
& y_{i j}=\left(t_{i j}^{\lambda}-1\right) / \lambda_{i i} \text { if } \lambda_{i} \neq 0, \quad y_{i j}=\log \left(t_{i j}\right) \text { if } \lambda_{i}=0, \\
& y_{i j}=x_{i j}{ }^{\prime} \beta+u_{i j} \quad i=1,2, \ldots, k, \quad j=1,2, \ldots, n_{i}
\end{aligned}
$$

where $t_{i j}$ is LOS, $\lambda$ is the transformation parameter, and $\beta$ are the $\mathrm{k}$-th dimensional vectors of the explanatory variables and coefficients, $k$ is the number of hospitals, $n_{i}$ is the number of patients in hospital 
Nawata and Kawabuchi, Evaluation of financial sustainability of the Japanese medical system: Analysis of the length of hospital stay for diabetes patients

$i$, and $n=\sum_{i} n_{i}$ respectively. $u_{i j}$ is assumed to follow the normal distribution with mean 0 and variance $\sigma_{i}^{2}$. Let $\theta^{\prime}=\left(\lambda, \beta^{\prime}, \sigma^{2}\right)$. The $\mathrm{BC}$ likelihood function under normality assumption of the error terms is given by

$$
\log L(\theta)=\sum_{t}\left[\log \phi\left\{\left(z_{t}-x_{t}{ }^{\prime} \beta\right) / \sigma\right\}-\log \sigma\right]+(\lambda-1) \sum_{t} \log y_{t},
$$

where $\phi$ is the probability density function of the standard normal assumption and $\sigma^{2}$ is the variance of $u_{t}$ under homoscedasticity. The BC MLE cannot be consistent generally even if the error terms are homoscedastic.

\subsection{A consistent estimator under heteroscedasticity}

Here, we assume $\lim _{N \rightarrow \infty} \bar{\sigma}_{n}^{2}=\bar{\sigma}^{2}>0$ where $\bar{\sigma}_{n}^{2}=\frac{\sum_{i, j} \log \left(\lambda_{0} x_{i j}{ }^{\prime} \beta_{0}+1\right) \sigma_{i}^{2}}{\sum_{i . j} \log \left(\lambda_{0} x_{i j}{ }^{\prime} \beta_{0}+1\right)}$. Instead of the BC MLE, we considered the roots of the equations,

$$
\begin{aligned}
& G(\theta)=\sum_{i, j}\left[-\frac{1}{\sigma^{2} \lambda}\left[\left\{\frac{\log \left(\lambda x_{i j}{ }^{\prime} \beta+1\right)}{\lambda}+\frac{z_{i j}-x_{i j}{ }^{\prime} \beta}{\lambda x_{i j}{ }^{\prime} \beta+1}\right\} y_{i j}^{\lambda}-z_{i j}\right\}\right]\left(z_{i j}-x_{i j}{ }^{\prime} \beta\right) \\
& \left.+\frac{1}{\lambda} \log \left(\lambda x_{i j}{ }^{\prime} \beta+1\right)+\frac{z-x_{i j}{ }^{\prime} \beta}{\lambda x_{i j}{ }^{\prime} \beta+1}\right] \equiv \sum_{i, j} g_{i j}(\theta)=0, \\
& \sum x_{i j}\left(z_{i j}-x_{i j}{ }^{\prime} \beta\right)=0, \\
& \text { and } H(\theta)=\sigma^{2}-\frac{\sum_{i, j} \log \left(\lambda_{0} x_{i j}{ }^{\prime} \beta_{0}+1\right)\left(z_{i j}-x_{i j}{ }^{\prime} \beta\right)^{2}}{\sum_{i . j} \log \left(\lambda_{0} x_{i j}{ }^{\prime} \beta_{0}+1\right)}=0 .
\end{aligned}
$$

Here,

$$
\begin{aligned}
G\left(\vartheta_{0}\right)= & -\frac{1}{\sigma^{2} \lambda_{0}} \sum_{i, j}\left[\left\{\frac{\left(\lambda_{0} x_{i j}{ }^{\prime} \beta_{0}+1\right) \log \left(\lambda_{0} x_{i j}{ }^{\prime} \beta_{0}+1\right)}{\lambda_{0}}-x_{i j}{ }^{\prime} \beta\right\} u_{i j}+\log \left(\lambda_{0} x_{i j}{ }^{\prime} \beta_{0}+1\right) u_{i j}^{2}+\frac{\lambda_{0} u_{i j}^{3}}{\lambda_{0} x_{i j}{ }^{\prime} \beta_{0}+1}\right] \\
& +\sum_{i, j}\left\{\frac{1}{\lambda_{0}} \log \left(\lambda_{0} x_{i j}{ }^{\prime} \beta_{0}+1\right)+\frac{u_{i j}}{\lambda_{0} x_{i j}{ }^{\prime} \beta_{0}+1}\right\}
\end{aligned}
$$

where $\vartheta_{0}^{\prime}=\left(\lambda_{0}, \beta_{0}, \sigma^{2}\right)$. Therefore, $E\left[\left.\frac{\partial \log L}{\partial \lambda}\right|_{\bar{\vartheta}_{n}}\right]=0$ where $\bar{\vartheta}_{n}=\left(\lambda_{0}, \beta_{0}, \bar{\sigma}_{n}^{2}\right)$. From the same argument of Nawata [8], there exists a consistent estimator of $\lambda$ and $\beta$ among the roots of Equation (4). Let $\hat{\theta}_{N}{ }^{\prime}=\left(\hat{\lambda}_{N}, \hat{\beta}_{N}, \hat{\sigma}_{N}^{2}\right)$ be the consistent root (hereafter, N-estimator) and $\bar{\vartheta}_{0}^{*}=\left(\lambda_{0}, \beta_{0}, \bar{\sigma}^{2}\right)$. As the homoscedastic cases, the asymptotic distribution of this estimator $\hat{\vartheta}_{n}$ is given by

$$
\sqrt{T}\left(\hat{\theta}_{N}-\vartheta_{0}^{*}\right) \rightarrow N\left[0, A^{-1} B\left(A^{\prime}\right)^{-1}\right]
$$

where

$$
A=-\lim _{n \rightarrow \infty} \frac{1}{n} \sum_{i, j} E\left[\left.\frac{\partial \xi_{i j}}{\partial \theta^{\prime}}\right|_{\vartheta_{0}^{*}}\right]
$$

$$
\xi_{i j}(\theta)^{\prime}=\left[-G(\theta), x_{i j}\left(y_{i j}-x_{i j}^{\prime} \beta\right), H(\theta)\right], B=-\lim _{n \rightarrow \infty} \frac{1}{n} \sum_{i, j} E\left[\left.\left.\frac{\partial \varsigma_{i j}}{\partial \theta}\right|_{\vartheta_{0}^{*}} \frac{\partial \varsigma_{i j}}{\partial \theta^{\prime}}\right|_{\vartheta_{0}^{*}}\right] \text {, }
$$


Nawata and Kawabuchi, Evaluation of financial sustainability of the Japanese medical system: Analysis of the length of hospital stay for diabetes patients

$\varsigma_{i j}\left(\theta_{0}\right)^{\prime}=\left[-G\left(\vartheta_{0}\right), x_{i j}{ }^{\prime} u_{i j}, w_{i}\left(u_{i j}^{2}-\sigma_{i}^{2}\right)\right]$, and $w_{i j}=\log \left(\lambda_{0} x_{i j}{ }^{\prime} \beta_{0}+1\right) /\left(\sum_{i . j} \log \left(\lambda_{0} x_{i j}{ }^{\prime} \beta_{0}+1\right) / n\right)$.

$\sigma_{i}^{2}$ is estimated by $\sigma_{i}^{2}=\sum_{j}\left(\hat{z}_{i j}-x_{i j}^{\prime} \hat{\beta}\right)^{2} / n_{i}$ where $\hat{z}_{i j}=\left(y_{i j}^{\hat{\lambda}}-1\right) / \hat{\lambda}$.

\section{ANALYSIS OF THE LENGTH OF STAY OF DIABETIC PATIENTS}

\subsection{Data}

In this section, we analyze the LOS of type 2 diabetic patients by the proposed model. The data set was collected from 86 hospitals by the Section of Health Care Economics, Tokyo Medical and Dental University. The survey period was from July 2008 to December 2008. For each patient, the DPC code, dates of hospitalization and discharge from the hospital, date of birth, sex, placement after hospitalization, ICD-10 code for the principle disease, purpose of hospitalization, presence of secondary disease and attending treatment if any, and medical payment amounts (including DPC-based, fee-for-service, and total payments) were reported. We analyze the data of the type 2 diabetic patients (DPC code: 100070xxxxxx0x). The per diem inclusive payments determined by the DPC/PDPS were 2,547 points for days 1-7 of hospitalization, 1,855 points for days $8-15$, and 1,577 points for 16-29 days in 2009 (Since 1958, 10 yen per point has been paid to hospitals). The total number of patients was 3,229 in 67 hospitals. We first excluded patients i) in clinical departments that do not treat diabetes, ii) whose principle disease was not diabetes, and ii) for whom the disease that caused hospitalization was not diabetes. We then used a data set of 1,571 patients in 17 hospitals (Hp1-17) having 60 or more patients.

Table 1 shows the LOS by hospitals. The average length of stay (ALOS) was 15.9 days, the median was 14.0 days, the standard deviation was 9.1 days, the skewness was 1.86, and the kurtosis was 9.04 (the kurtosis is the value where the normal distribution is 0 ). The maximum ALOS by hospital was 24.6 days (Hp10) and the minimum ALOS was 10.8 days (Hp7). The maximum was 2.3 times larger than the minimum, and the difference was 13.8 days. The maximum standard deviation by hospital was 12.0 days (Hp10), and the minimum was 5.3 days (Hp8), which was less than one-third of the maximum. The variances were quite different among hospitals, even for hospitals whose ALOSs were similar. This implies the importance of the proposed model, which takes into account the heteroscedasticity of the variances. Finally, the skewness and kurtosis values were large for some of the hospitals. In other words, the distributions for these hospitals differed from the normal distribution: the large skewness and kurtosis values for certain hospitals imply that some patients remained in these hospitals for a long period of time.

Table 1. LOS by hospitals.

\begin{tabular}{|r|r|r|r|r|r|}
\hline Hospital & ALOS & $\begin{array}{r}\text { Standard } \\
\text { deviation }\end{array}$ & Hospital & ALOS & $\begin{array}{r}\text { Standard } \\
\text { deviation }\end{array}$ \\
\hline Hp1 & 14.9 & 3.9 & Hp10 & 24.6 & 5.0 \\
\hline Hp2 & 17.0 & 4.1 & Hp11 & 15.9 & 4.0 \\
\hline Hp3 & 23.7 & 4.9 & Hp12 & 24.3 & 4.9 \\
\hline Hp4 & 11.6 & 3.4 & Hp13 & 13.3 & 3.7 \\
\hline Hp5 & 16.5 & 4.1 & Hp14 & 15.7 & 4.0 \\
\hline Hp6 & 17.3 & 4.2 & Hp15 & 16.9 & 4.1 \\
\hline Hp7 & 10.8 & 3.3 & Hp16 & 12.7 & 3.6 \\
\hline Hp8 & 14.7 & 3.8 & Hp17 & 11.2 & 3.4 \\
\hline Hp9 & 16.8 & 4.1 & All & 15.9 & 9.1 \\
\hline
\end{tabular}

\subsection{Results of Estimation}

We chose the following variables as explanatory variables. The Female Dummy (0: male, 1 : female) was used for gender. The proportions of male and female patients were $59.5 \%$ and $40.5 \%$, respectively. Since the LOS tends to increase with patient age, we use Age as an explanatory variable. The average age of the patients was 62.2 , and its standard deviation was 13.63. Among diabetic patients, there are patients whose purpose is to join educational programs for managing diabetes at home rather than receiving regular medical treatments. For the purpose of hospitalization, we used the Education Dummy (joining educational program: 
Nawata and Kawabuchi, Evaluation of financial sustainability of the Japanese medical system: Analysis of the length of hospital stay for diabetes patients

1. otherwise: 0 ). The proportion of patients joining educational programs was $43.2 \%$. Other explanatory variables representing the characteristics of patients included Secondary Diseases (numbers of secondary diseases), Complications (numbers of complications), Acute Hospitalization Dummy (acute hospitalization: 1, otherwise: 0), Outpatient Dummy (outpatient of the same hospital before hospitalization: 1, otherwise: 0), and Other Hospital Dummy (1: discharged to another hospital or facility: 1, otherwise: 0 ). Among our study subjects, $78.5 \%$ of patients had secondary diseases, and among those with secondary diseases the average number was 2.23 . A total of $29.9 \%$ of patients had complications, and those patients had 2.00 complications on average. The proportions of the acute hospitalization patients, outpatients of the same hospital before hospitalization, and patients discharged to another hospital or facility were $15.7 \%, 90.8 \%$, and $33.9 \%$, respectively.

For principal disease classification, dummy variables based on the ICD-10 code E119 were used. For classification, $32.1 \%$ of patients had diseases classified under E119, 5.9\% had diseases under E112, 5.5\% had diseases under E113, 7.9\% had diseases under E114, 0.8\% had diseases under E115, 13.2\% had diseases under E116, and 33.9\% had diseases under E117. Nawata and Kawabuchi (2015) reported that many patients were discharged from hospitals on the eighth day (after one week of hospitalization) and fifteenth day (after two weeks of hospitalization); hence, we added Day 8 (discharged on the eight day: 1, otherwise: 0 ) and Day 15 Dummies (discharged on the fifteenth day: 1, otherwise: 0 ), and found that $6.4 \%$ and $6.2 \%$ of patients left on the eighth and fifteenth days, respectively. Furthermore, if the LOS exceeded the Specific Hospitalization Period (29 days), the medical payment became the conventional fee-for-service system. Therefore, we added the Over-Specific-Period Dummy (LOS over 29 days: 1, otherwise: 0 ), and found that $8.1 \%$ of patients stayed over 29 days. As the result, of Equation (5) becomes

$$
\begin{aligned}
& x_{i j}{ }^{\prime} \beta_{1}=\beta_{1} \text { Female Dummy }+\beta_{2} \text { Age }+\beta_{3} \text { Secondary Diseases }+\beta_{4} \text { Complications } \\
& +\beta_{5} \text { Acute Hospitalization Dummy }+\beta_{6} \text { Education Dummy }+\beta_{7} \text { Outpatient Dummy } \\
& +\beta_{8} \text { Other Hospital Dummy }+\beta_{9} \text { Day } 8 \text { Dummy }+\beta_{10} \text { Day } 8 \text { Dummy } \\
& +\beta_{11} \text { Over Specific Period Dummy } \sum_{\ell} \beta_{\ell} \ell \text {-th Principle Disease Dummy }
\end{aligned}
$$

The estimation results are presented in Table 2. There exist large differences in the estimates of $\sigma_{i}$, and the feasibility of the proposed model was strongly suggested. The estimation results of Equation (4) are given in Table 3. The estimates of Inspection and Educational Dummies are negative and significant at $1 \%$ and $5 \%$ levels; that implies the LOS became shorter if the purpose of hospitalization was not medical treatment. The estimates of the Secondary Diseases and Complications were positive and significant at the $1 \%$ level. This means that the secondary diseases and complications made the LOS longer, as expected. The estimates of Day 8 and Over-Specific-Period Dummies were positive at the 1\% level, and one-week hospitalization and specific hospitalization period affected LOS longer. The estimates for Age, Female, Acute Hospitalization, Education, Outpatient, Other Hospital, and Day 15 Dummies were not significant at the 5\% level, and we could not find any evidence that the LOS depends on these variables. With respect to the principal disease classifications, the estimate for the E117 Dummy was negative and significant at the 5\% level, but none of the other estimates was significant at the $5 \%$ level.

To evaluate the effects of the ALOS by hospitals it is necessary to consider the changes in the characteristics of patients. If differences of ALOS among hospitals are caused by changes in the characteristics of patients, we cannot say that ALOS differs among hospitals. Here, we analyzed the LOS while controlling for the patient characteristics. Table 3 represents the ALOS of hospitals for a patient who is male, age 60, no complications or secondary diseases, hospital outpatient, not acute hospitalization, purpose of hospitalization is regular medical treatment, returns home, and principle disease is E119. We found surprisingly large differences among hospitals, with the longest ALOS, 14.6, being more than double the shortest, 6.9 days. If the ALOS of all hospitals were reduced to 6.9 days, ALOS weighted by the numbers of patients by hospitals could be reduced by 3.6 days. The DPC payment was 18,550 yen per day and the average medical expenditure per type 2 diabetes patient was 329,078 yen. Therefore, $20.4 \%$ of medical expenditure for diabetes patients could be saved. In our dataset, the total medical expenditure including all types of diabetes was 1,479 million yen and the expenditure for type 2 diabetes patients was 1,062 million comprising $71.8 \%$ of the expenditure. The total medical expenditure for diabetes with hospitalization was 320 billion yen in the fiscal year 2012. This means that we could save as much as 50 billion yen by this revision. 
Nawata and Kawabuchi, Evaluation of financial sustainability of the Japanese medical system: Analysis of the length of hospital stay for diabetes patients

Table 2. Results of estimation.

\begin{tabular}{|c|c|c|c|c|c|c|c|c|}
\hline Variable & Estimate & $\begin{array}{l}\text { Standard } \\
\text { error }\end{array}$ & $t$-value & Variable & Estimate & $\begin{array}{l}\text { Standard } \\
\text { error }\end{array}$ & $t$-value & $\sigma_{i}$ \\
\hline & 0.9395 & 0.0102 & $91.739^{* *}$ & \multicolumn{5}{|c|}{ Hospital Dummies } \\
\hline $\begin{array}{l}\text { Female } \\
\text { Dummy }\end{array}$ & 0.1479 & 0.2338 & 0.632 & Hp1 & 9.4675 & 0.8650 & $10.945^{* *}$ & 3.3083 \\
\hline Age & 0.0086 & 0.0092 & 0.933 & $\mathrm{Hp} 2$ & 12.2779 & 0.9399 & $13.064^{* *}$ & 3.6144 \\
\hline $\begin{array}{l}\text { Inspection } \\
\text { Dummy }\end{array}$ & -7.6102 & 0.6265 & $-12.148^{* *}$ & Hp3 & 13.5651 & 0.8651 & $15.681^{* *}$ & 3.9600 \\
\hline $\begin{array}{l}\text { Education } \\
\text { Dummy }\end{array}$ & -0.7732 & 0.3067 & $-2.521^{*}$ & Hp4 & 7.4479 & 0.9532 & $7.814^{* *}$ & 2.7953 \\
\hline Complications & 0.6843 & 0.1358 & $5.038^{* *}$ & Hp5 & 10.5738 & 0.8761 & $12.069^{* *}$ & 3.4741 \\
\hline $\begin{array}{l}\text { Secondary } \\
\text { Diseases }\end{array}$ & 0.5860 & 0.1254 & $4.672^{* *}$ & Hp6 & 10.5609 & 0.9273 & $11.389^{* *}$ & 3.3747 \\
\hline $\begin{array}{l}\text { Acute } \\
\text { Hospitalization }\end{array}$ & 0.4037 & 0.4625 & 0.873 & Hp 7 & 6.6213 & 0.9670 & $6.847^{* *}$ & 2.6167 \\
\hline $\begin{array}{l}\text { Other Hospital } \\
\text { Dummy }\end{array}$ & 0.1256 & 0.3033 & 0.414 & Hp8 & 9.6426 & 0.9186 & $10.497^{* *}$ & 3.2398 \\
\hline $\begin{array}{l}\text { Outpatient } \\
\text { Dummy }\end{array}$ & -0.2528 & 0.4737 & -0.534 & Hp9 & 12.9564 & 0.9186 & $14.104^{* *}$ & 3.7555 \\
\hline \multicolumn{4}{|c|}{ Principle disease Dummies } & Hp10 & 13.1955 & 1.0151 & $13.000^{* *}$ & 3.6055 \\
\hline E112 & -0.2571 & 0.5390 & -0.477 & Hp11 & 10.7303 & 0.8432 & $12.725^{* *}$ & 3.5673 \\
\hline E113 & -0.2680 & 0.4695 & -0.571 & Hp12 & 13.8941 & 0.9797 & $14.183^{* *}$ & 3.7660 \\
\hline E114 & 0.1769 & 0.4134 & 0.428 & Hp13 & 9.3533 & 0.7595 & $12.315^{* *}$ & 3.5093 \\
\hline E115 & 4.2812 & 2.2846 & 1.874 & Hp14 & 10.8027 & 0.9610 & $11.241^{* *}$ & 3.3528 \\
\hline E116 & 0.2791 & 0.4443 & 0.628 & Hp15 & 10.2886 & 0.9048 & $11.371^{* *}$ & 3.3720 \\
\hline E117 & 0.7637 & 0.3353 & $2.278^{*}$ & Hp16 & 8.3713 & 0.8169 & $10.248^{* *}$ & 3.2012 \\
\hline Day 8 Dummy & -4.0360 & 0.3958 & $-10.197^{* *}$ & Hp17 & 7.6858 & 0.8762 & $8.772^{* *}$ & 2.9617 \\
\hline $\begin{array}{l}\text { Day } 15 \\
\text { Dummy } \\
\end{array}$ & -0.3045 & 0.2841 & -1.072 & \multirow[b]{2}{*}{$R^{2}$} & \multirow{2}{*}{\multicolumn{4}{|c|}{0.6452}} \\
\hline $\begin{array}{l}\text { Over Specific } \\
\text { Period } \\
\text { Dummy }\end{array}$ & 16.9792 & 1.2210 & $13.906^{* *}$ & & & & & \\
\hline
\end{tabular}

*: Significant at the 5\% level, **: Significant at the $1 \%$ level.

Table 3. The ALOS while controlling for patient characteristics by hospital.*

\begin{tabular}{|l|r|l|r|l|r|}
\hline Hospital & \multicolumn{1}{|l|}{ ALOS } & Hospital & \multicolumn{1}{|l|}{ ALOS } & Hospital & \multicolumn{1}{l|}{ ALOS } \\
\hline Hp1 & 9.7 & $\mathrm{Hp} 7$ & 6.9 & $\mathrm{Hp} 13$ & 9.6 \\
\hline Hp2 & 12.5 & $\mathrm{Hp} 8$ & 9.9 & $\mathrm{Hp} 14$ & 11.1 \\
\hline Hp3 & 13.8 & $\mathrm{Hp} 9$ & 13.2 & $\mathrm{Hp} 15$ & 10.6 \\
\hline Hp4 & 7.7 & $\mathrm{Hp} 10$ & 13.4 & $\mathrm{Hp} 16$ & 8.6 \\
\hline Hp5 & 10.8 & $\mathrm{Hp} 11$ & 11.9 & $\mathrm{Hp} 17$ & 7.9 \\
\hline Hp6 & 10.8 & $\mathrm{Hp} 12$ & 14.6 & Average & 10.7 \\
\hline
\end{tabular}

*: for a patient who is male, age 60, no complications or secondary diseases, hospital outpatient, not acute hospitalization, purpose of hospitalization is regular medical treatment, returns home, and principle disease is E119. 
Nawata and Kawabuchi, Evaluation of financial sustainability of the Japanese medical system: Analysis of the length of hospital stay for diabetes patients

\section{CONCLUSION}

In this paper, we consider an analysis of the LOS by the power Box-Cox transformation model when variances differ among hospitals and the number of hospitals increases to infinity. We propose a new consistent estimator. We also obtained its asymptotic distribution. We found large differences in the estimates of transformation parameters and variances among hospitals, and the feasibility of the proposed model was strongly suggested. Finally, we analyzed the LOS of 1,571 type 2 diabetes patients collected from 17 general hospitals in Japan. The variables found to affect the LOS were the numbers of secondary diseases and complications, one-week hospitalization, specific hospitalization period and principal disease E117.

Since the introduction of the DPC in Japan, medical information has been computerized in many hospitals. Data obtained from more than a thousand DPC hospitals will be available for analysis in the near future. To evaluate and control medical expenditures in Japan more precisely, it is necessary to analyze data sets of this size. It is also necessary to analyze other important case types such as cancer to evaluate the sustainability of the Japanese medical insurance system. These are subjects to be analyzed in future studies.

\section{ACKNOWLEDGEMENTS}

This study was supported by a Grant-in-Aid for Scientific Research, "Analyses of Large Scale Medical Survey Data and Policy Evaluations in Japan (Grant Number: 24330067)," from the Japan Society of Science. The Institutional Review Boards of the University of Tokyo and Tokyo Medical and Dental University approved the use of this dataset.

\section{REFERENCES}

[1] Box, G. E. P., and D. R. Cox (1964), “An Analysis of Transformation,” Journal of the Royal Statistical Society B, 26, 211-252.

[2] Hossain, M. Z. (2011) "The Use of Box-Cox Transformation Technique in Economic and Statistical Analyses," Journal of Emerging Trends in Economics and Management Sciences 2, 32-39.

[3] Ministry of Health, Labour and Welfare (2014), Hesiei 24 nendo kokumin iryouhi (National Medical Expenditures, Fiscal Year 2012, in Japanese).

[4] Mutou, K., Y. Uchigata, and H. Yamada, 1999, "18 sai miman hasshou IDDM kanja nonyuuin nissu ni kansuru chousa (Investigation on Hospitalization Days in Crisis of IDDM Child Patients under 18 Years Old)," Purakutisu (Journal of Practical Diabetes), 16:302-306 (in Japanese).

[5] Kudo, T, Y. Fujino, A. Matsuura et al. (2011), "Prevalence of type 2 Diabetes among acute Inpatients and its impact on length of hospital stay in Japan Diabetes mellitus and the risk of cancer: results from a large-scale population-based cohort study in Japan,” Internal Medicine, Vol. 50, 405-411.

[6] Inoue M., M. Iwasak, T. Otani et al. (2006), "Diabetes mellitus and the risk of cancer," Archives of Interna-tional Medicine, 166, 1871-1877.

[7] Nawata, K., M. Ii, H. Toyama et al. (2009), "Evaluation of the inclusive payment system based on the Diagnosis Procedure Combination with respect to cataract operations in Japan - A comparison of lengths of hospital stay and medical payments among hospitals," Health, Vol.1, No.2, 93-103.

[8] Nawata, K. "A new estimator of the Box-Cox transformation model using moment conditions," 2013, Economics Bulletin, Vol. 33, Issue 3, 2287-2297.

[9] Nawata. K. and K. Kawabuchi, (2015), "Evaluation of Length of Hospital Stay Joining Educational Programs for Type 2 Diabetes Mellitus Patients: Can We Control Medical Costs in Japan?" Health, 7, 256-269.

[10] Sakia, R. M. (1992), “The Box-Cox Transformation Technique: A Review,” The Statistician, 41, 169178.

[11] Showalter, M. H. (1994), "A Monte Carlo Investigation of the Box-Cox Model and a Nonlinear Least Squares Alternative," Review of Economics and Statistics, 76, 560-570.

[12] Sohmiya M., S. Yonehara, M. Sumikawa, et al. (2004) "Tounyoubyou no nyuuin nissuu ni eikyou wo oyobosu youin ni kansuru kentou (Review of the Factors Influencing the Diabetic Patients' LOS in the Hospital)," Rinsyou to Kenkyuu (The Japanese Journal of Clinical and Experimental Medicine), 81, 1031-1033 (in Japanese). 